\title{
Visualizando oportunidades educativas mediante las TIC
}

\section{Visualizing Educational Opportunities Through ICTs}

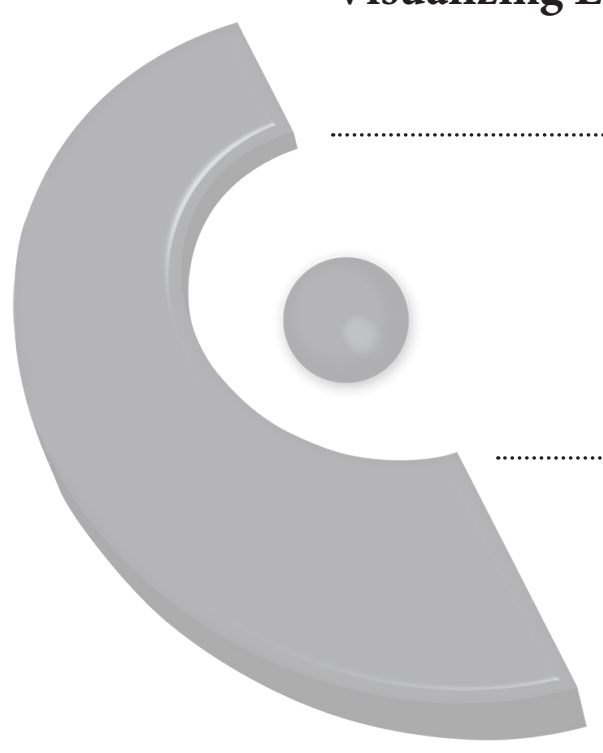

\author{
Marlene Durán López \\ Universidad Nacional \\ Heredia, Costa Rica \\ marlene.duran.lopez@una.cr
}

Recibido 22/05/2017 Aceptado 30/08/2017

Resumen. Las buenas prácticas pedagógicas conllevan a los centros de enseñanza a concebir el tema tecnológico de forma responsable, ética y equitativa. Pensar en la generación de aprendizaje de forma integral conlleva a visualizar la tecnología no como el fin, sino como el medio para lograr los objetivos propuestos. Entender las nuevas tendencias tecnológicas, desde una mirada crítica y pertinente, abre un panorama que se traduce en oportunidades para el crecimiento integral de todos los actores involucrados en el proceso de enseñanza y aprendizaje. Ante este panorama, surge el cuestionamiento tal como lo manifiesta Lévy:

¿Cómo mantener las prácticas pedagógicas de acuerdo con estos nuevos procesos de transacción de conocimiento? Aquí no se trata de utilizar a todo precio las tecnologías sino de acompañar consciente y deliberadamente un cambio de civilización que vuelve a cuestionar profundamente las formas institucionales, las mentalidades y la cultura de los sistemas educativos tradicionales y, sobre todo, los roles del profesor y del alumno (2007, p. 144). 
Revista Universidad en Diálogo • Vol. 7, N. 2, Julio-Diciembre, 2017, pp. 25-36

ISSN 2215-2849 • EISSN: 2215-4752

DOI: http://dx.doi.org/10.15359/udre.7-2.2

Estos nuevos roles se presentan ante los nuevos escenarios tecnológicos, y han de ser concebidos dentro de una estrategia que brinde igualdad de oportunidades y faculte a los actores a mantener la cultura e identidad ante la globalización y las barreras que presenta una sociedad de información ante una sociedad de conocimiento.

Palabras clave: TIC, brecha digital, e-learning, m-learning.

Abstract. Good teaching practices help schools to conceive technology in a responsible, ethical and equitable way. Thinking of the generation of learning in a holistic way leads to the visualization of technology, not as the end, but as the means to achieve objectives. Understanding the new technological trends from a critical and relevant perspective opens a panorama that translates into opportunities for integral growth of all the people involved in the process of teaching and learning. We might ask the question proposed by Lévy (2007):

How can we keep our teaching practices in a way that meets the requirements of these new processes of transaction knowledge? It is not a question of using technology at any cost, but of accompanying, consciously and deliberately, a civilization change that will deeply challenge, again, institutional forms, mentalities, and the culture of traditional education systems and, above all, the roles of the teacher and the student. These new roles are presented in the context of new technology scenarios and should be designed within a strategy providing equal opportunities that will empower actors to maintain the culture and identity in front of the globalization and the barriers an information society presents to a knowledge society.

Keywords: information and communication technologies, digital divide, e-learning, m-learning.

La incorporación de las tecnologías de información y comunicación (TIC) en el ámbito educativo ha sido concebida como una necesidad, tras esfuerzos que han logrado potenciar el uso estratégico de la tecnología en busca de nuevas fuentes de generación de conocimiento. Los nuevos ambientes educativos del siglo XXI han revolucionado los métodos tradicionales de enseñar y aprender, y esto ha dado como resultado políticas públicas que garantizan el acceso y la equidad de la educación como un pilar del progreso de las sociedades, al ser concebidos como derechos de la humanidad.

Un aporte significativo de las TIC a las sociedades ha sido el fortalecer y ampliar el acceso a la educación, al permitir a sectores de la población con desigualdad de condiciones -sean estas económicas, circunstanciales, 
geográficas o de exclusión- tener una oportunidad, pues permiten el acceso a la tecnología desde su casa, centro educativo o espacios públicos.

La influencia que ejercen las TIC en la motivación del estudiantado es positiva y estimula la creatividad y el interés; supera los medios y barreras tradicionales, gracias a la interactividad, la multimedia y entornos virtuales que proporcionan herramientas, tales como los simuladores. Un simulador le presenta a la persona usuaria un escenario con situaciones o espacios que, en condiciones reales, sería difícil conocer o utilizar, y, a su vez, representan una oportunidad previa para enfrentar las situaciones en su contexto real.

Un viaje a un museo o sitios históricos, un experimento, el ensamble de un dispositivo son ejemplos prácticos de simuladores virtuales, abren las puertas de las aulas tradicionales a un mundo de posibilidades por descubrir.

Estos nuevos ambientes han dado paso a la educación virtual, tras exponer sus beneficios y traspasar las fronteras producto de la globalización. El acceso a la educación en cualquier parte del mundo es posible gracias a nuevas metodologías de enseñanza, como el e-learning, donde la presencia física del estudiantado no es un elemento característico del entorno educativo.

Esta modalidad es posible gracias a la tecnología que proporciona la plataforma para lograr el producto esperado y responde auna oportunidad para que cualquier estudiante, no importa su ubicación geográfica o situaciones particulares, acceda a un conocimiento específico o a herramientas que le faculten para mejorar su calidad de vida. Es posible cursar exitosamente carreras, postgrados y especialidades y aprovechar las ventajas que conlleva traspasar los límites de un déficit en la oferta educativa local.

Aprender con estas nuevas tecnologías supone un cambio en los paradigmas tradicionales y sus cuestionamientos, demanda un aprender y un desaprender ante la dinámica constante del entorno y ante las modalidades emergentes, que demandan un papel más preponderante del estudiantado en la búsqueda y acceso al conocimiento, así como del personal docente en las estrategias pedagógicas para lograr fortalecer y aplicar el concepto de aprendizaje para la vida.

Una particularidad positiva de las TIC es su potencial para propiciar el aprendizaje colaborativo, creando un espacio de interacción que gira en torno a un interés en común, o a distintas áreas del saber que promueven una intervención activa de los sujetos participantes, facilitando la asimilación de conceptos e involucrando a personal experto que actúe como guía del aprendizaje. 
Revista Universidad en Diálogo • Vol. 7, N. 2, Julio-Diciembre, 2017, pp. 25-36

ISSN 2215-2849 • EISSN: 2215-4752

DOI: http://dx.doi.org/10.15359/udre.7-2.2

Estos espacios pueden ser aprovechados por diversas dinámicas que involucren la participación de los sujetos aprendientes, desde cualquier dispositivo tecnológico tan pequeño como un celular hasta uno más extenso como un laboratorio o una pizarra digital interactiva. Surge aquí un tema de oportunidad y acceso que se traduce en las posibilidades que un centro pueda tener para adquirir el equipo necesario que permita trabajar y aprovechar sus ventajas al máximo.

Cabe recalcar que el uso de la tecnología como una herramienta pedagógica requiere una inversión que responda a las necesidades propias del contexto. Así, muchas veces, su adquisición, sin una guía pedagógica y participación activa del personal docente, se traduce solo en adquisición tecnológica. La figura 1 permite observar que, en un entorno tecnológico, se puede aprender de muchas maneras y el rol que desempeñe cada participante es esencial para lograr la asimilación del saber.

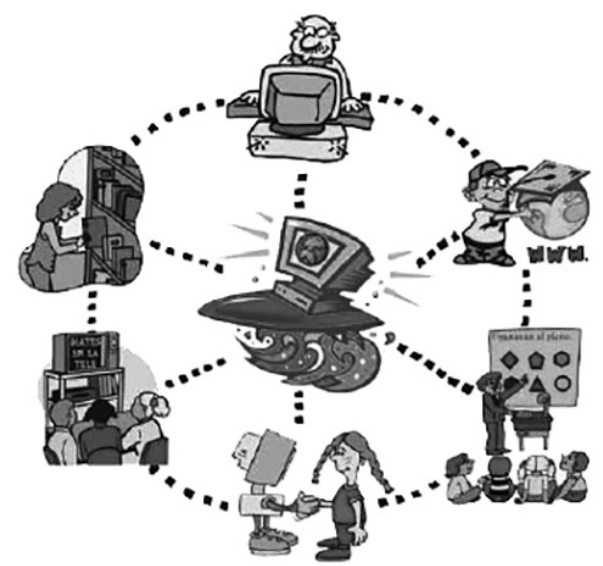

Figura 1. Aprendizaje mediante la tecnología (Figueroa, 2011)

Es importante notar que estos ambientes no están limitados al uso exclusivo de una computadora, más bien conforme avanza la tecnología, nuevos dispositivos y herramientas emergen y vienen a formar parte de los recursos a emplear, depende de la creatividad y dedicación que se disponga para el asunto.

Como parte del dinamismo y las estrategias comerciales que han surgido en el mercado, nacen contrapartes para lograr que los recursos en software propietario tengan su equivalente en software libre y, de este modo, ampliar las posibilidades de un acceso equitativo y ético de la tecnología. Cabe también mencionar que, homólogamente a la adquisición de equipo de alto costo, 
como por ejemplo una pizarra digital interactiva, existe su versión limitada a un costo inferior, como lo es un dispositivo Leap Motion, que ofrece muchas funcionalidades, pero a su vez más limitadas que las de la pizarra digital.

El Leap Motion es un dispositivo pequeño que conectado a una computadora permite controlar la interfaz del sistema operativo a través de gestos, lo cual lo convierte en un recurso sumamente llamativo y a la vez económico. $\mathrm{Su}$ implementación en el salón de clases o en cualquier espacio es fácil, y la potencialidad para lograr generar conocimiento solo depende de las estrategias y la creatividad para llevar a cabo una clase o actividad completamente diferente.

Estas tecnologías mencionadas presentan, ante el público usuario, una gama de posibilidades de lo que se puede lograr cuando se cuenta con el recurso tecnológico, o se adquiere tecnología económica que, adaptada a una computadora o complementada con otro dispositivo, puede lograr efectos y funcionalidades mayores.

En contraste con lo anterior, cabe mencionar un tema que desde tiempos antiguos y a través de las décadas ha permanecido en mayor o menor medida: las brechas en el acceso a la enseñanza. Recapitulando en el tiempo, el acceso a la educación fue garantizado solo a cierto grupo que poseyera los recursos que le permitieran acceder a dicho privilegio. Con el pasar de los años, barreras y paradigmas se han ido superando, para que mayor número de personas accedan a una educación digna y en igualdad de condiciones.

Estos esfuerzos, aunque no han sido en vano, no han logrado los resultados esperados y nuevas brechas surgen producto del progreso y de la globalización. Es de esperar que la mayoría de personas reconozcan en la educación un canal para mejorar la calidad de vida $\mathrm{y}$, por ende, pretendan el acceso a los centros de enseñanza. Esta dinámica global presenta, al mismo tiempo, una brecha generacional entre quienes nacieron con la tecnología y quienes no. Esta desventaja requiere un doble esfuerzo para aquellas personas que requieren reaprender sus prácticas tradicionales por medio de las nuevas formas que expone el uso de la tecnología.

Esta limitación en el orden cronológico, a su vez, ha desencadenado una brecha de pensamiento, que podemos notar en aquellas personas que se resisten a utilizar el potencial tecnológico para lograr los objetivos propuestos y se limitan a un uso básico de este. Así mismo, podemos observar una brecha en el pensamiento de aquellas personas que utilizan las tecnologías de información y comunicación en diversas formas, pero no para un uso efectivo que traduzca los datos e información en conocimiento. 
Revista Universidad en Diálogo • Vol. 7, N. 2, Julio-Diciembre, 2017, pp. 25-36

ISSN 2215-2849 • EISSN: 2215-4752

DOI: http://dx.doi.org/10.15359/udre.7-2.2

Según La Nación, "en el 94\% de los hogares costarricenses hay, por lo menos, un teléfono celular y la penetración de Internet crece a ritmo acelerado" (2014, párr. 1). Esta estadística refleja datos significativos que se pueden aprovechar para canalizar la tecnología hacia las aulas e incentivar en los usuarios su uso para aprender.

Otro dato significativo lo presenta El Financiero, donde se alude a que "Costa Rica tiene 1,5 líneas celulares por habitante" (Cordero, 2014). Estas cifras, comparadas con el siguiente dato: "a penetración del servicio celular (151\%) coloca al país como el mayor usuario de esta tecnología en Centroamérica y cerca de países como Uruguay (155\%) y Singapur (156\%)", son alertas hacia las cuales se debe desviar la mirada, para lograr que estos dispositivos tecnológicos se conviertan en aliados, que sean utilizados para disminuir brechas que otros recursos tecnológicos hayan generado.

Según Muñoz y Nicaragua (2013. p.21):

Lasuniversidades tienen un papel importante quejugar,pues son llamadas a ser agentes de cambio en favor del desarrollo social y solidario; por lo tanto, sus planes de estudio y sus proyectos de investigación deberían primero incluir el fortalecimiento de las competencias digitales de los estudiantes, más allá de los aspectos operacionales y, en segundo lugar, desarrollar investigación que permita plasmar nuevos modelos de uso e implementación de las TIC en cualquier ámbito.

Para respaldar la cita anterior, menciono una nueva modalidad de aprendizaje conocida como mobile learning (m-learning), que, como los datos estadísticos han mencionado, se puede aprovechar particularmente, dada la penetración del dispositivo celular en la sociedad costarricense y, en general, a nivel mundial.

El m-learning se concibe como el uso de dispositivos móviles para el apoyo docente y es una modalidad que ha tenido muchas críticas, desencadenando dos bandos claramente definidos: el que apoya esta metodología de aprendizaje, potencializando su uso, y el que abiertamente se opone a incorporar su uso en el salón de clases como una herramienta pedagógica.

Según La Nación, referente al tema del uso de los celulares en clase:

La mediación del profesor como guía de aprendizaje permite que el celular deje de ser el enemigo y se convierta en un aliado en el diseño de trabajos cotidianos y tareas. De esa forma los estudiantes aprenden a usar correctamente la herramienta que tienen en su poder (2016, párr. 6). 
Esta no es una tarea fácil, ya que se debe tener en cuenta una serie de factores, entre los que se incluye la conectividad y la guía docente para lograr el aprendizaje propuesto, así como el romper paradigmas ya arraigados.

El Ministerio de Educación Pública emitió su directriz al respecto: "El uso de dispositivos móviles se realizará en las actividades específicamente preparadas por los docentes y las docentes y según el planeamiento de clase previamente establecido" (MEP, 2016, p. 5). En consecuencia, de no haber una mediación pedagógica, estos dispositivos serán un distractor más en el ambiente de aprendizaje y un obstáculo para la asimilación correcta del contenido.

Como lo muestra la figura 2, el aprendizaje a través de tecnologías móviles es una construcción y está en "construcción", dado que su implementación aún no ha sido muy difundida, por la serie de factores que hay que tomar en cuenta para su exitosa inclusión en el proceso educativo del estudiantado.

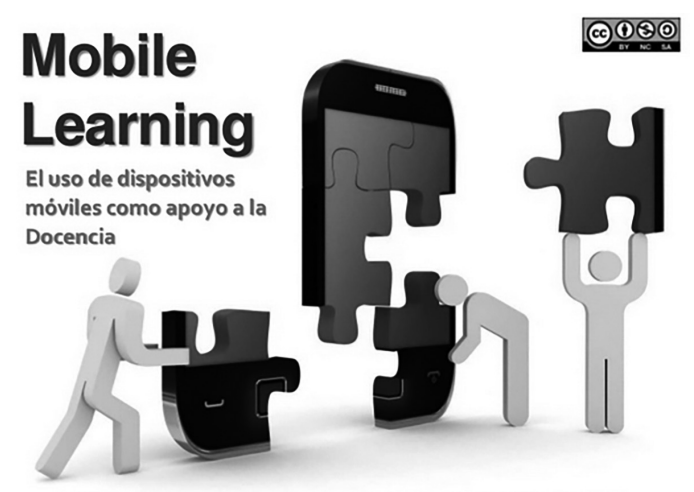

Figura 2. Mobile Learning (Marquina, 2013)

Esta construcción requiere tiempo, cambio de paradigmas, creatividad, esfuerzo y una guía pedagógica con regulaciones claras.

Ante estas nuevas oportunidades que emergen de la tecnología, las políticas públicas deben ir acompañadas de los recursos necesarios que proporcionen la favorable implementación de los diversos recursos tecnológicos y del seguimiento requerido para lograr que las nuevas modalidades educativas tengan éxito ante los contextos cambiantes y dinámicos de la sociedad. Dichas propuestas requieren un compromiso de todos los actores involucrados para lograr las metas y proyectos en torno a la educación pública. 
Consecuentemente, es necesaria la preparación del recurso humano para enfrentar estas nuevas estrategias pedagógicas y es fundamental dinamizar los aportes y potencialidades de las herramientas tecnológicas hacia un empoderamiento que permita visualizar propuestas y soluciones a demandas educacionales, con una participación activa de autoridades tomadoras de decisiones, docentes, personal administrativo y estudiantes que intervienen en el proceso de enseñanza y aprendizaje, para un abordaje integral que responda a las demandas de las situaciones particulares, con la pertinencia y las consideraciones sociales y culturales del caso.

Para que todas estas estrategias y metas se lleven a cabo debe haber una planificación que permita la incorporación, evaluación y seguimiento para la consolidación de una cibercultura, que garantice la continuidad y mantenimiento de las prácticas en el tiempo. También dentro de las líneas de acción, comprende el principio de oportunidades para el acceso y equidad en zonas marginadas, que debe traducirse en la disminución de la desigualdad y no en una nueva forma de marginación conocida como brecha digital.

Según datos del Consejo Nacional para Investigaciones Científicas y Tecnológicas (CONICIT), el Valle Central acapara los índices de mayor acceso a los recursos tecnológicos.

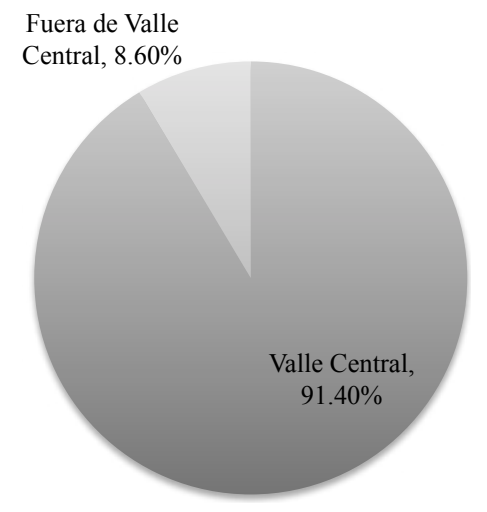

Figura 3. Distribución de la telefonía y el internet (Vargas y Espinoza, 2012).

Nota. Encuesta realizada por CINPE-Proyecto REINA (abril-junio, 2012). 
Estas estadísticas revelan, a quienes toman las decisiones, índices importantes para decidir acciones concretas que se tornen en igualdad de condiciones para todas las personas que acceden a una educación pública de calidad.

Según las Políticas de las TIC y Educación (2006), presentadas en el seminario Políticas en TIC y Educación para Centroamérica, la brecha digital debe considerarse desde la perspectiva de tres factores interrelacionados: conectividad, acceso y equidad. La conectividad se vincula a la infraestructura; el acceso con la capacidad de adquisición; y la equidad directamente con las políticas públicas que permiten universalizar el acceso a los servicios, involucrando zonas vulnerables o aquellas que están en desventaja, como las comunidades rurales.

De todo lo anterior podemos desprender retos y desafíos que no son fáciles de llevar a cabo, pero con una visión de futuro integral se pueden convertir en acciones concretas que dinamicen el potencial tecnológico al servicio de la educación.

Resulta una necesidad la inclusión de la tecnología en los centros educativos en igualdad de condiciones y pertinencia, que responda a los desafíos que la sociedad de información y conocimiento demanda de las instituciones educativas.

De igual forma, es un requerimiento el promover el uso de la tecnología desde actitudes críticas y éticas, que utilicen las tecnologías de información y comunicación para potenciar eficientemente los procesos educativos y la adquisición del conocimiento.

Por consiguiente, es importante que desde el sector educacional se siembre y promueva el interés por aprender y reaprender ante la dinámica tecnológica que trae consigo herramientas altamente potenciales para su aprovechamiento en los diversos entornos y ambientes educacionales. Como resultado, se propone una apertura y actualización constante, para formar parte de los procesos que conllevan las exigencias de un mercado globalizado.

Según la figura 4, tomada de la red social Pinterest, la palabra educar incluye, por sí misma, una serie de tareas y retos, los cuales, soportados con recursos tecnológicos, pueden traducirse en una educación para la vida. 
Revista Universidad en Diálogo • Vol. 7, N. 2, Julio-Diciembre, 2017, pp. 25-36

ISSN 2215-2849 • EISSN: 2215-4752

DOI: http://dx.doi.org/10.15359/udre.7-2.2

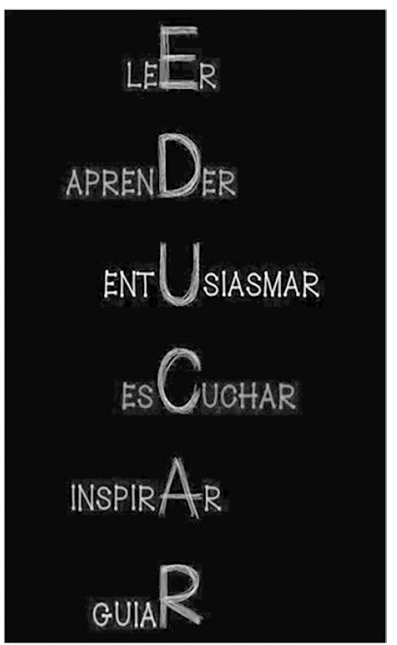

Figura 4. Educar (Pinterest, 2014).

Siendo la educación un camino infinito de saberes, surge la siguiente interrogante: ¿Hacia dónde vamos? Vamos hacia entornos más extensos y dinámicos: la realidad virtual y la computación cognitiva. Si bien es cierto que estos conceptos fueron asociados a las películas de cine en ciencia ficción, hoy representan una realidad que se desarrolla en las aulas de nuestros centros universitarios, donde la investigación ha dado paso para que la tendencia popular hacia nuevos entornos pueda ser canalizada para una experiencia educativa sin fronteras, que vincule la educación, la tecnología y la realidad virtual en un espacio tan pequeño o tan grande como lo logre abarcar su imaginación.

El mercado ha revelado que los costos y las opciones de adquisición son muchas y que estas pueden variar de acuerdo con las posibilidades de adquisición de los diversos centros, pero que cualquiera que sea el recurso a utilizar debe estar claramente soportado con una estrategia general que garantice la generación del conocimiento.

Hoy la educación no se concibe sin tecnología, y, como podemos ver en la siguiente afirmación, muchos han sido los esfuerzos a nivel mundial por lograr llevar a cabo, en forma conjunta, soluciones que integren propuestas de diversos países hacia una sociedad comprometida con su educación: 
Los principales retos educativos que enfrenta la región de Centroamérica, al igual que otros países y regiones, giran en torno a las demandas formativas derivadas de la sociedad y economía del conocimiento y al compromiso de las naciones por dar cumplimiento a los objetivos y acuerdos del Foro Mundial de Educación firmado en Dakar, Senegal en abril del 2000 "Educación Para Todos" (EPT) (Políticas de TIC y Educación, 2006, p. 14).

Estos son solamente algunos elementos de los desafíos de la educación para fundar las bases de una sociedad de información y conocimiento, que enriquezca su cultura y que sea incluyente en todas las sociedades, cualquiera que fuera su condición, con actores activos que aporten creativamente en un mundo, más que generador de información, productor de conocimiento.

\section{Referencias}

Cordero, M. (30 de julio, 2014). Costa Rica tiene 1,5 líneas celulares por habitante. El financiero. Recuperado de http://www. elfinancierocr.com/tecnologia/Costa-Rica-lineas-celulareshabitante_0_564543547.html

Figueroa, G. (2011). Aprendizaje colaborativo y TIC. Recuperado de https://aprendizajecolaborativo1.wordpress.com/page/2/

La Nación. (12 de noviembre, 2014). Aumento en la conectividad. La Nación. Recuperado de http://www.nacion.com/opinion/editorial/ Aumento-conectividad_0_1450854916.html

La Nación. (02 de marzo, 2016). MEP pretende convertir el celular en un aliado en las aulas. La Nación. Recuperado de http://www.nacion. com/nacional/educacion/MEP-celulares-aulas_0_1546045478.html

Lévy, P. (2007). Cibercultura: la cultura de la sociedad digital. México: Anthropos Editorial.

Marquina, R. (2013). Situación actual del M-learning en Venezuela. Recuperado de $\mathrm{http}$ :/es.slideshare.net/raymarq/situacin-actual-del-mlearning-en-venezuela

MEP. (2016). Lineamientos generales para el uso de dispositivos móviles propiedad de los estudiantes en el centro educativo. Recuperado de http://www.mep.go.cr/sites/default/files/descargas_etica/circular.pdf 
ReVista Universidad en Diálogo • Vol. 7, N. ${ }^{\circ}$ 2, Julio-Diciembre, 2017, pp. 25-36

ISSN 2215-2849 • EISSN: 2215-4752

DOI: http://dx.doi.org/10.15359/udre.7-2.2

Muñoz, M. y Nicaragua, R. (2013). Un acercamiento a la brecha digital en Costa Rica desde el punto de vista del acceso, la conectividad y la alfabetización digital. e-Ciencias de la Información, 4(1). Recuperado de http://revistas.ucr.ac.cr/index.php/eciencias/article/ view/12866/12469

Pinterest. (2014). Frases educación [red social]. Recuperado de https:// es.pinterest.com/explore/frases-educacion/

Políticas de TIC y Educación. (2006). Conectividad, acceso y equidad. Seminario de Políticas en TIC y Educación para Centroamérica.

Vargas, L. y Espinoza, J. (2012). Telefonía e internet en Costa Rica. Recuperado de http://www.conicit.go.cr/ver/biblioteca/ publicaciones/publicacionescyt/otros_documentosCyT/ TELEFONIA_E_INTERNET.pdf\#.WR8n9dxuPIU 